Die sinnvolle und möglichst korrekte Messung von Behandlungsergebnissen gilt als die grösste und wahrscheinlich schwierigste Herausforderung in der Palette des Qualitätsnachweises von medizinischen Behandlungen. Die Stiftung EQUAM, die sich seit Jahren intensiv mit Qualitätsfragen in der ambulanten Leistungserbringung auseinandersetzt, wagt sich mit neuen Modulen der Qualitätsmessung und des -nachweises an dieses Thema. Erste Versuche mit neuen klinischen Standards zeigen das grosse Potential solcher Instrumente und lassen einen wirklichen Gewinn in der Behandlungsqualität von bestimmten Patientengruppen vermuten.

Um die Einstiegshürde in solche doch recht aufwendige Zertifizierungsbemühungen etwas weni- ger hoch zu gestalten, hat man einen Ansatz gewählt, der es jedem Einzelnen ermöglicht, sich langsam in die Themen einzuarbeiten. Es gilt aber nicht nur, sich ständig methodologisch zu verbessern, sondern auch die Anreize im Gesundheitswesen so zu setzen, dass sich der Aufwand für solche Bemühungen, im Sinne der verbesserten $\mathrm{Pa}$ tientenversorgung, lohnt. Hier sieht die FMH eine ihrer Kernaufgaben darin, auf dem politischen Parkett so einzuwirken, dass Anreizsysteme geschaffen werden, die eine solche sinnvolle Qualitätsarbeit anerkennen und honorieren.

Dr. med. Daniel Herren, MHA, Mitglied des Zentralvorstandes der FMH, Verantwortlicher für das Ressort DDQ

\title{
Zwei markante Meilensteine in der ambulanten Qualitätsförderung
}

\author{
Nach mehrjähriger Entwicklungsarbeit kann die Stiftung EQUAM ${ }^{1}$ drei Zertifizie- \\ rungssets mit Indikatoren zur Performance-, Ergebnis- und Indikationsqualität vor- \\ weisen. Erstmals haben sich nun im Sommer 2009 drei Grundversorger einem Zerti- \\ fizierungsaudit zu den Minimal Standards der Diagnose Diabetes mellitus unterzo- \\ gen und dazu die innovative Softwareeigenentwicklung Diabetes Databox des \\ Ärztenetzes mediX zürich genutzt.
}

Kurt Hess, Marco Vecellio

1 EQUAM (Externe Qualitätssicherung in der Medizin): unabhängige, vom Bund (seco) akkreditierte Stiftung zur Praxiszertifizierung.

Korrespondenz:

Dr. med. et lic. oec. Kurt Hess Grossmünsterplatz 1

CH-8001 Zürich

kurt.hess@hin.ch
Die Erkrankung Diabetes mellitus Typ 2 ist weltweit auf dem Vormarsch. So werden für das Jahr 2025 in der Schweiz durch die WHO approximativ $600000 \mathrm{Er}$ krankte prognostiziert. Wir wissen aus zahlreichen Studien, dass durch konsequente präventive und interdisziplinäre Betreuungsansätze Stoffwechseleinstellung, Ernährungsgewohnheiten und Lebensqualität verbessert werden können. Ebenfalls lassen sich dadurch die Anzahl an Hospitalisationen wegen Diabetes und dessen Komplikationen wie Herzinfarkt, Hirnschlag, Erblindung, Amputationen oder Niereninsuffizienz signifikant reduzieren. Erfahrungen aus anderen Ländern zeigen zudem, dass internetbasierte Entscheidungshilfen bei erfolgreicher Integration in den Praxisalltag die Betreuungsqualität günstig beeinflussen.

Valide epidemiologische Daten zur ambulanten Betreuung von Diabetes-Patienten in schweizerischen Hausarztpraxen sind - ganz im Gegensatz zu vielen anderen Ländern - allerdings bis anhin noch kaum verfügbar. Umgekehrt weisen Entwicklungen in der medizinischen Qualitätssicherung sowie politische Forderungen zunehmend auf die zentrale Bedeutung der Ergebnis- und Performancequalität hin, wobei der bisherige Fokus aus verschiedenen Gründen einseitig auf der Struktur- und Prozessebene lag. EQUAM und mediX zürich haben sich diesen Postulaten und Entwicklungen gemeinsam gestellt und - mangels verfügbarer Systeme von Softwareanbietern - eigene Lösungen in Zusammenarbeit mit weiteren Institutionen erarbeitet.

\section{Ein Durchbruch im Qualitätsnachweis - klinische Indikatoren und Standards}

Seit ihrer Gründung 1999 hat die Stiftung EQUAM darauf hingearbeitet, eine möglichst breite Abstützung im Stiftungsrat aufzubauen. Heute haben alle Akteure im Gesundheitswesen Einsitz - in Kürze auch die FMH - und garantieren nicht nur die Unabhängigkeit der Institution, sondern führen einen offenen und fruchtbaren Dialog, der im Grossen und auf höheren Ebenen nicht gelingen will (s. Kasten Stiftungsrat). 2006 hat der Stiftungsrat den Auftrag zur Erarbeitung von klinischen Indikatoren erteilt, um den allerorts geäusserten Forderungen zu entsprechen und damit das bisherige Zertifizierungsinstrumentarium zu ergänzen.

Das Set an Indikatoren und Standards ist modular aufgebaut und besteht aus drei Teilen: 


\section{Der EQUAM-Stiftungsrat}

(* Mitglieder des Ausschusses)

Simonetta Sommaruga*, Präsidentin, Mitglied des Ständerates (SP), Präsidentin Stiftung für Konsumentenschutz

Dr. med. Ignazio Cassis, MPH, Vizepräsident FMH, Mitglied des Nationalrates (FDP)

Dr. med. Massimo Ermanni, SUVA, Leiter Agenturärztlicher Dienst VMA

Dr. iur. Gebhard Eugster, ehemaliger Ombudsmann der sozialen Krankenversicherung

Dr. med. Daniel Herren, MHA, Facharzt Orthopädie, Leitung Daten, Demographie und Qualität im FMH Zentralvorstand (Wahlantrag in der Stiftungsratssitzung vom 16.11.2009)

Dr. med. et lic. oec. Kurt Hess*, Vizepräsident, Berater im Gesundheitswesen

PD Dr. med. Ludwig T. Heuss, M.B.A. HSG, Chefarzt Medizin, Spital Zollikerberg

Dr. med. Felix Huber*, Ärztlicher Leiter medix zürich

Margrit Kessler, Präsidentin Schweiz. Patientenorganisation SPO

Dr. iur. et lic. phil. I Niklaus Lüchinger, Rechtsanwalt Verena Nold Rebetez, mag. oec. HSG, Stv. Direktorin santésuisse, Leiterin Abteilung Verträge und Tarife, Präsidentin TARMED Suisse

Dr. med. Florian Suter, praktizierender Hausarzt, Geschäftsführer Ärztenetz Nordwest

Erika Ziltener, lic. phil. I, Historikerin, Pflegefachfrau, Präsidentin der Patientenstelle Zürich und des Dachverbandes Schweizerische Patientenstellen DVSP, Kantonsrätin Zürich

- Basismodul A: Standards auf der Grundlage des European Practice Assessment (EPA) ${ }^{2}$ und in enger Zusammenarbeit mit Swisspep ${ }^{3}$

- Zusatzmodul B: Höherwertige Minimal Standards für Versorgungsmodelle des Managed Care (Ärztenetzwerke und HMO-Praxen)

- Freiwilliges klinisches Zusatzmodul C: diagnosebezogene Standards der Ergebnis-, Performance- und Diagnosequalität.

2 European Practice Assessment (EPA), international validiertes und europaweit etabliertes Indikatorensystem für ambulante Praxen, in der Schweiz betrieben durch Swisspep

3 Swisspep Institut für Qualität \& Forschung im Gesundheitswesen, Bern

4 AQUA-Institut für angewandte Qualitätsforschung in Göttingen (D)

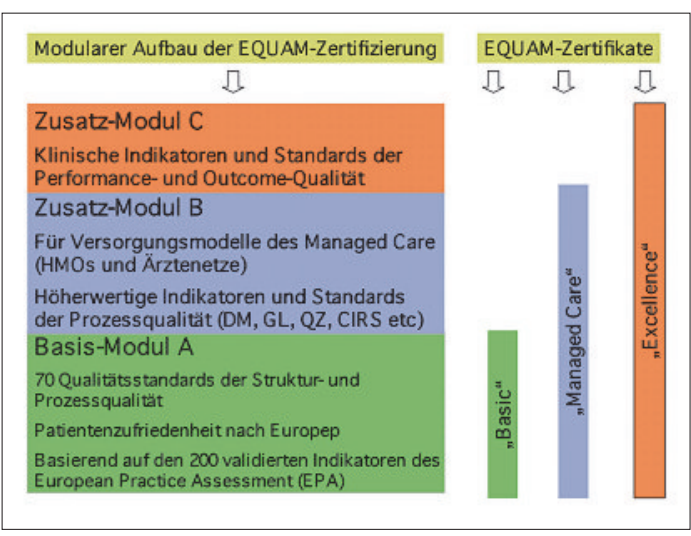

Was den klinischen Qualitätsnachweis betrifft, so liegen seit Mitte 2009 die ersten beiden Module zur arteriellen Hypertonie und zum Diabetes mellitus vor und sind bereits vom Bund (seco) akkreditiert worden. Standards für eine dritte Diagnose, Koronare Herzkrankheit, sind in enger Zusammenarbeit mit Swisspep und der internationalen Studie EPA Cardio in Entwicklung und stehen kurz vor der Fertigstellung. Als vierte Entwicklung im Bereich der Outcome- und Performancequalität prüft EQUAM gegenwärtig in Zusammenarbeit mit der argomed Ärzte AG und der SUVA die Erarbeitung eines Moduls, mit dem sich die Qualität der ärztlichen Arbeitsfähigkeitsbeurteilung evaluieren lässt. Weitere klinische Module - so zu Asthma, Rückenschmerzen und Medikationssicherheit - sind für die kommenden zwei, drei Jahre geplant.

Im letzten Juli sind nun erstmals in der Grundversorgung drei Zertifizierungsaudits mit den neuen klinischen Indikatoren und Standards durchgeführt worden. Drei Ärzte des Ärztenetzes mediX zürich haben sich den EQUAM-Standards zum Diabetes mellitus gestellt und den Nachweis ihrer Ergebnis- und Performancequalität zu dieser Diagnose erbracht. Geführt und begleitet hat diesen Audit Prof. Dieter Conen. In seinem Bericht an die Schweizerische Akkreditierungsstelle, deren Fachexperte er ist, weist Prof. Conen auf die Evidenzbasierung sowohl der Indikatoren wie von deren Validierung hin und betont, dass sich bei Einhaltung dieser Indikatoren die Prognose der Diabetespatienten nach den relevanten Studien verbessert. Auf der Basis dieses Berichts und nach Prüfung der Zertifikationsunterlagen ist dieses Modul akkreditiert worden. Der hierbei erstmalige Einbezug von Indikatoren der Ergebnis- und Indikationsqualität in der ambulanten Betreuung von Diabetikern darf als markanter Meilenstein im Bereich der ambulanten Qualitätsförderung gelten.

Zwei wesentliche Grundlagen haben diesen Durchbruch geprägt: Zum einen hat EQUAM diese Entwicklung nicht allein zuwege bringen können und wollen, sondern kooperiert seit Jahren mit massgeblichen schweizerischen und internationalen Institutionen der Qualitätsförderung: Swisspep, EPA, AOK-Bundesverband, AQUA-Institut ${ }^{4}$, mediX und anderen Ärztenetzwerken. Auch der Erfahrungsaustausch mit den Exponenten des britischen National Health Service (NHS) und mit der deutschen Stiftung Praxissiegel hat die Entwicklungsarbeit mitgeprägt.

\section{Diabetes Databox - eine Eigenentwicklung}

Eine zweite Conditio sine qua non, welche die Dokumentation der Diabetesparameter mit praktikablem Aufwand erst ermöglicht hat, ist die Softwareentwicklung Databox. Die Praktikabilität von Ergebnisindikatoren setzt die Vollständigkeit und Korrektheit der Dokumentation in den Patientenakten voraus, was nur durch die digitale Erfassung effizient umsetzbar ist. Die arrivierten Softwareunternehmen haben es ver- 


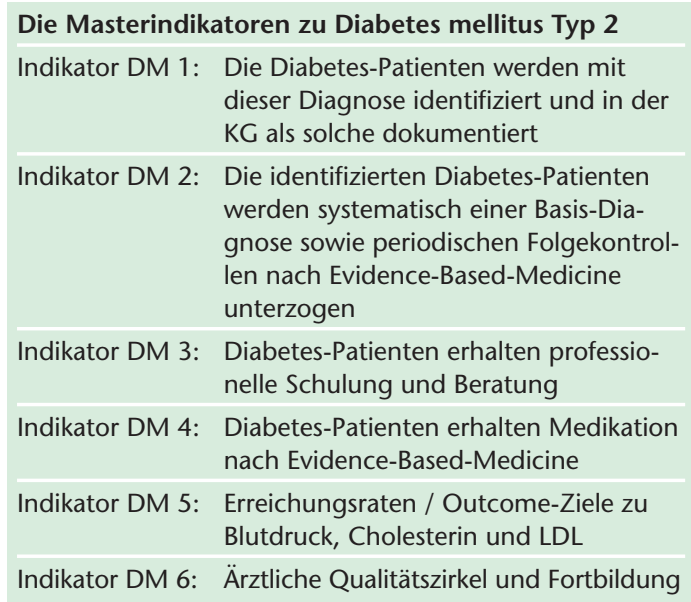

säumt, dafür Lösungen zu entwickeln, weshalb Dr. med. Marco Vecellio im Auftrag von mediX zürich die webbasierte Datenbanklösung Databox in enger Zusammenarbeit mit CIRS Software von Grund auf neu entwickelt hat, die eine flexible Erfassung, Dokumentation, Auswertung sowie die gruppenspezifische Analyse verschiedener Indikatoren ermöglicht.

Es lassen sich Laborwerte, klinische Befunde, vereinbarte Zielsetzungen, medikamentöse Therapien sowie Patient-Empowerment-Strategien protokollieren. Ausserdem sind statistische Auswertungen und extensive Exportfunktionen integriert. Vertraulichkeit der Daten, kontinuierliche Verfügbarkeit und Integrität wurden durch den Einsatz moderner Datenbanktechnologie sichergestellt. Der angeschlossene Hausarzt zieht aus gezielten Feedbacks, Remindinghilfen, Ampelsystemen und lokalen Statistischen Auswertungen für seine Patientenbetreuung direkten Nutzen.

Die Anzahl der regelmässig mit der Databox erfassenden Ärzte und Ärztinnen im Netz beträgt heute rund 20\%. Ende August 2009 wurden 564 Patienten eingeschlossen, welche sich auf 30 aktiv beteiligte Ärzte aufteilen. Nach Überprüfung der Datensätze wurden insgesamt 1896 Einzelkontrollen durchgeführt und in der Datenbank regulär erfasst. Der mittlere Beobachtungszeitraum je Patient betrug seit Projektbeginn bis dato 356 Tage.

Aus der Analyse dieser Verlaufsdaten und der Praktikabilität dieses Projektes konnten wichtige Einsichten zur Umsetzung und Formulierung von klinisch relevanten und validierten Indikatoren zur Outcomeund Performancequalität im Bereich der Diabetesversorgung im schweizerischen Praxisalltag gewonnen werden. Erstmals haben wir hiermit Einblick in wichtige Aspekte der ambulanten Betreuungsqualität bei Diabetikern gewonnen. Auswertungen werden in Fachzeitschriften und am Wonca-Kongress präsentiert.

\section{Der weite Weg in die Zukunft}

Die breite Ärzteschaft steht dem Postulat, ihre Arbeit und deren Ergebnisse - auch und vor allem für Patienten - transparent zu machen, nach wie vor skeptisch gegenüber. Mit einigem Recht hat sie stets darauf hingewiesen, dass die gängigen Struktur- und Prozessindikatoren für eine relevante Qualitätsbeschreibung nicht genügen können. Diesem Argument fehlt nun mit der Entwicklung von Outcome- und Performanceindikatoren zunehmend die Überzeugungskraft.

Mit den vorgestellten «Primeurs» sind die Wege der Weiterentwicklung klar vorgezeichnet. Eine zunehmende Zahl von Ländern weist uns zudem den Weg. Auf der Ebene der Qualitätsinstrumentarien folgen die periodischen Aktualisierungen von Indikatoren und Standards sowie die erwähnte Ergänzung des klinischen Moduls um weitere Diagnosen. Auf Stiftungsebene stehen die Festigung der bestehenden Kooperationen sowie das Eingehen weiterer Partnerschaften an. EQUAM sieht sich immer noch gewissen Berührungsängsten gegenüber, die allerorts still und leise entstehenden Eigengewächse zeugen davon. Diese permanente Neuerfindung des Rades generiert gewaltige Kosten und völlig inkompatible Systeme, nur eine Bündelung der Kräfte kann zielführend sein. Und auf politischer Ebene suchen wir Wege, im Interesse der Patientensicherheit neue Anreize zu schaffen: Wer seine Qualität - als Netzwerk wie auch als Arzt oder Ärztin - nachweist, soll anders honoriert werden als derjenige, der dies verweigert.

\section{Weiterführende Literatur}

- American Diabetes Association. Standards of Medical Care in Diabetes. Diabetes Care. 2008;(31) Suppl. 1.

- IDF Clinical Guidelines Task Force. Global Guideline for Type 2 Diabetes. Brussels: International Diabetes Federation; 2005. http://www.idf.org

- Guidelines on diabetes, pre-diabetes, and cardiovascular diseases. The Task Force on Diabetes and Cardiovascular Diseases of the European Society of Cardiology (ESC) and of the European Association for the Study of Diabetes (EASD) European Heart Journal. 2007;(28):88-136.

- National Collaborating Centre for Chronic Conditions. Type 2 diabetes: national clinical guideline for management in primary and secondary care (update) London: Royal College of Physicians; 2008.

- Tuomiletho J, Lindstroem J, Eriksson JG, Valle TT, Hämäläinen $H$, Ilanne-Parikka $P$, et al. Prevention of type 2 diabetes mellitus by changes in lifestyle among subjects with impaired glucose tolerance. N Engl J Med. 2001;344(18):1343-50.

- Knight K, Badamgarav E, Henning JM, Hasselblad V, Gano AD, Ofman JJ, et. al. A Systematic Review of Diabetes Disease Management Programs. The American Journal of Managed Care. 11:242-50.

- Meigs J, Chueh H, Cagliero E, Barry M, Dubay A, Singer A, et. al. Controlled Trial of Web-Based Diabetes Disease Management. Diabetes Care. 2003;26:750-7. 